\title{
Efficacy of biologic therapies for biologic-naïve Japanese patients with moderately to severely active ulcerative colitis: a network meta-analysis
}

\author{
Toshifumi Hibi ${ }^{1}$, Isao Kamae $^{2}$, Philippe Pinton ${ }^{3}$, Lyann Ursos ${ }^{4}$, Ryuichi Iwakiri ${ }^{3}$, Greg Hather ${ }^{5}$, Haridarshan Patel ${ }^{6 *}$ \\ ${ }^{1}$ Center for Advanced IBD Research and Treatment, Kitasato University Kitasato Institute Hospital, Tokyo, ${ }^{2}$ Department of Health Policy and \\ Technology Assessment, Graduate School of Public Policy, The University of Tokyo, Tokyo; ${ }^{3}$ Japan Medical Office, Takeda Pharmaceutical \\ Company Limited, Tokyo, Japan; ${ }^{4}$ Global Medical Affairs, Takeda Pharmaceuticals USA, Inc., Deerfield, IL; ${ }^{5}$ Statistical \& Quantitative \\ Sciences, Takeda Pharmaceuticals USA, Inc., Cambridge, MA; ${ }^{6}$ Department of Evidence and Value Generation, Global Medical Affairs, Takeda \\ Pharmaceuticals USA, Inc., Deerfield, IL, USA
}

Background/Aims: Several biologic therapies are approved in Japan to treat moderately to severely active ulcerative colitis (UC), but there are no published comparative efficacy studies in a Japanese population. We compared the efficacy of biologics approved in Japan (adalimumab, infliximab, golimumab, and vedolizumab) for treating biologic-naïve patients with UC at their approved doses. Methods: A targeted literature review identified 4 randomized controlled trials of biologics for UC in biologicnaïve Japanese patients. For each study, efficacy outcome data from induction (weeks 6-12) and maintenance (weeks 30-60) treatment were extracted for analysis. Treatment effects on clinical response, clinical remission, and mucosal healing relative to the average placebo results across all trials were estimated using network meta-analyses followed by transformation into probabilities and odds ratios (OR). Results: At the end of induction, the likelihood of clinical response and clinical remission was highest with infliximab (OR: 2.12 and 2.35, respectively) and vedolizumab (OR: 2.10 and 2.32, respectively); the likelihood of mucosal healing was highest with infliximab (OR: 2.24) and adalimumab (OR: 1.86). During maintenance, the likelihood of clinical response and clinical remission was highest with vedolizumab (OR: 6.44 and 4.68, respectively) and golimumab (OR: 5.13 and 3.84, respectively); the likelihood of mucosal healing was significantly higher than placebo with all biologics. Conclusions: All active treatments were efficacious compared with placebo. Infliximab and vedolizumab had the highest odds for induction of clinical response, remission, and mucosal healing. Golimumab and vedolizumab had numerically higher odds of achieving efficacy outcomes in the maintenance phase. (Intest Res 2021;19:53-61)

Key Words: Biologics; Colitis, ulcerative; Network meta-analysis; Efficacy

\section{INTRODUCTION}

Ulcerative colitis (UC) is a chronic, relapsing, inflammatory disease of the colon characterized by alternating episodes of

Received November 21, 2019. Revised February 5, 2020

Accepted March 2, 2020.

Correspondence to Toshifumi Hibi, Center for Advanced IBD Research and Treatment, Kitasato University Kitasato Institute Hospital, 5-9-1 Shirokane, Minato-ku, Tokyo 108-8462, Japan. Tel: +81-3-5791-6487, Fax: +81-33357-6156,E-mail: thibi@insti.kitasato-u.ac.jp

*Haridarshan Patel was an employee of Takeda at the time of this research. This study was presented as a poster at the 2019 Crohn's \& Colitis Congress (CCC) on February 7-9, 2019, in Las Vegas, NV, USA. remission and spontaneous relapse. ${ }^{1}$ Patients with active UC experience significant and clinically meaningful impairment across most aspects of quality of life., ${ }^{2,3}$ The prevalence of UC in Japan has been steadily increasing from 18.2 in 1991 and 63.6 in 2005 to 121.9 per 100,000 persons in $20133^{4}$ about 170,000 patients were receiving treatment for UC in Japan in 2017.5

Aminosalicylates (5-ASAs) are used as first-line therapy for induction and maintenance of remission in mild to moderately active UC; patients who do not respond to 5-ASAs are treated with corticosteroids (second-line treatment), whereas immunomodulators and biologics are used in moderately to se- 
verely active UC. ${ }^{6}$ The introduction of biologics has changed the treatment paradigm for moderately to severely active UC. Currently, adalimumab, infliximab, and golimumab, which target tumor necrosis factor (TNF)- $\alpha$, and vedolizumab, which blocks recruitment of immune cells to the gut by targeting $\alpha_{4} \beta_{7}$ integrin, ${ }^{7}$ are approved to treat UC in Japan. ${ }^{8,9}$

The traditional primary treatment goal in UC has been the induction and maintenance of disease remission. However, the treatment goals for UC have evolved from resolution of UC symptoms to include objective measures such as mucosal healing. Consistent with this trend, the Selecting Therapeutic Targets in Inflammatory Bowel Disease consensus endorses the use of endoscopic outcomes as therapeutic goals in clinical practice. ${ }^{10}$

Population differences may be an important factor in meeting UC treatment goals. For example, differences in phenotypes of UC and susceptibility to UC due to genetic polymorphisms have been reported in different populations. ${ }^{11,12}$ As such, response to UC treatment may vary in different ethnic populations.

Direct head-to-head trials assessing the relative efficacies of biologics in biologic-naïve Japanese patients are lacking. Assessment of comparative efficacy and safety using network meta-analysis (NMA) can supplement, or compensate for the lack of, relevant randomized controlled trials (RCTs). ${ }^{13}$ In absence of formal direct head-to-head evidence, these methods can assist policymakers and healthcare professionals in evidence-based decision-making. NMA studies can provide indirect comparisons of treatment efficacy of biologic therapies in moderately to severely active UC and generate useful evidence for judiciously selecting the best choice(s) of treatment. In this study, using data from published RCTs, an NMA was conducted to indirectly compare treatment efficacies of biologics approved in Japan (adalimumab, infliximab, golimumab, and vedolizumab) for treating biologic-naïve patients with moderately to severely active UC.

\section{METHODS}

\section{Study Selection}

A targeted review of the literature was conducted to identity published articles or peer-reviewed, published scientific congress presentations reporting RCTs evaluating approved biologics in biologic-naïve Japanese patients with UC. PubMed and Embase were searched in February 2019 using the following terms: adalimumab, infliximab, golimumab, or vedolizum- ab, combined with UC. The full search strategies for PubMed and Embase are "(ulcerative colitis [Title] AND (adalimumab [Title/Abstract] OR golimumab [Title/Abstract] OR infliximab [Title/Abstract] OR vedolizumab [Title/Abstract])) AND (randomized [Title/Abstract] OR randomised [Title/Abstract])" and "ulcerative colitis.title. AND (vedolizumab or golimumab or infliximab or adalimumab). abstract. AND (randomized or randomised).abstract.", respectively. The search was limited to RCTs conducted in human subjects and no language restrictions were applied. Duplicate publications were excluded, and each unique publication identified in the searches was reviewed for relevance by title and abstract. The full text of the selected articles was retrieved and evaluated based on study eligibility criteria. Studies were eligible for inclusion if they were RCTs with more than one treatment arm and assessed the efficacy or safety of biologics for the treatment of biologicnaïve Japanese patients with moderately to severely active UC. Both placebo-controlled trials and active treatment-controlled trials were eligible for inclusion. Only studies that used approved dosing of each drug (as provided in the respective summary of product characteristics) and had a follow-up time of 1 year were included.

\section{Outcome Measures and Definitions}

Outcome measures of interest in the induction phase were clinical response, clinical remission, and mucosal healing. Induction endpoints were assessed at week 8 for infliximab and adalimumab and at week 10 for vedolizumab. Outcome measures of interest in the maintenance phase were sustained clinical response, sustained clinical remission, and mucosal healing at weeks 30 to 60 . Definitions of outcome measures in induction phase and maintenance phase are presented in Supplementary Table 1.

\section{Data Extraction}

All data were extracted by 2 independent reviewers and discrepancies were discussed and resolved. Details on the trial's acronym, first author's last name, year of publication, study design, and patient baseline characteristics were extracted from each publication. Number of participants and intervention parameters including drug, dosage, and administration were extracted separately for both induction phase and maintenance phase where available. Data were only collected for patients not previously exposed to anti-TNF therapy. Data for pre-specified outcome measures in biologic-naïve patients were extracted for each study at the end of treatment induc- 
tion and maintenance phases where available. Different dosages of the same treatment were treated as different interventions.

\section{Quality Assessment}

The risk of bias for each included study was evaluated using the Cochrane Collaboration tool for assessing risk of bias in RCTs. ${ }^{14}$ The following items were evaluated: generation of the allocation sequence (selection bias); concealment of the allocation sequence (selection bias); blinding (detection and performance bias); blinding of participants and personnel and blinding of outcome assessment; incomplete outcome data (attrition bias); selective outcome reporting (reporting bias); other biases (Supplementary Table 2). For each RCT, each item was evaluated as: low risk of bias, high risk of bias, or unclear risk of bias. ${ }^{14}$

\section{Data Synthesis and Analysis}

The treatment effect at the end of the induction phase and at 1 year for each biologic was estimated using Bayesian metaanalyses and was transformed into probabilities and odds ratios (ORs) with 95\% credible intervals (CrI) using the average placebo results across all trials as a reference. Risk differences were calculated as the differences in the probabilities of each efficacy outcome against placebo. Each of the pre-specified outcome measures was analyzed separately at each relevant time point (induction and maintenance). The analysis assumed that outcome measures were defined similarly between trials.

A binomial likelihood, logit link function, and fixed-effects model were used to account for variability between trials, treatment group, and treatment effect. For direct comparisons, a fixed-effects (as opposed to random-effects) model $^{15}$ was used due to the limited number of studies included in the analysis, which did not allow random-effects terms to be estimated.

Small-study effects and publication bias were not formally assessed, given that each pairwise comparison included a limited number of studies (less than 10). The analysis was performed using OpenBUGS version 3.2.2, following U.K. National Institute for Health and Care Excellence guidance on evidence synthesis methodology.

\section{Compliance with Ethical Standards}

As part of the original studies used as data sources for the current analyses, all patients provided written informed consent, and the trials were approved by the institutional review board of each participating institution. Because the current post hoc analyses used existing data from published primary studies, additional patient consent was not required.

\section{RESULTS}

\section{Study and Patient Characteristics}

Four phase 3 RCTs were included in the NMA based on the eligibility criteria; publication dates ranged from 2014 to 2019 . $^{16-19}$ Baseline study and patient characteristics are presented in $\mathrm{Ta}-$ ble $1 .{ }^{16-19}$ Sample sizes per study ranged from 186 to 246 patients in 3 studies reporting efficacy outcomes in the induction phase ${ }^{17-19}$ and from 63 to 273 patients in the maintenance phase in 4 studies. ${ }^{16-19}$ The mean age of patients ranged from 37.8 to 44.0 years across studies and treatment arms within individual studies. The percentages of male patients varied between studies and between treatment arms within individual studies (range, 51\%-73\%). The network of eligible comparisons for a treatment efficacy NMA is illustrated in Fig. 1.

\section{Study Quality}

Results using the Cochrane Collaboration tool indicated that the risk of bias in all categories was low, with the exception of bias based on the methods employed for allocation concealment; risk of bias for "allocation concealment" was unclear for all 4 studies. Taken together, the overall risk of bias in all 4 included studies was low (Supplementary Table 2).

\section{Induction Phase Efficacy Outcomes}

Treatment efficacy was examined using data from week 6 to week 12 in the 3 trials that reported outcomes following adalimumab, infliximab, and vedolizumab treatment during induction (Fig. 1A).

The likelihood of clinical response and remission with adalimumab was not significantly different from placebo. In contrast, compared with placebo, patients who received infliximab and vedolizumab had a higher probability of induction response and remission (Table 2). Odds of clinical response were significantly higher with infliximab (OR, 2.12; 95\% CrI, 1.26-3.54) and with vedolizumab (OR, 2.10; 95\% CrI, 1.044.42) (Fig. 2A).

Likewise, the odds of clinical remission were significantly higher with infliximab (OR, 2.35; 95\% CrI, 1.31-4.08) and with vedolizumab (OR, 2.32; 95\% CrI, 1.05-5.16) (Fig. 2B). Compared with placebo, the probability of mucosal healing was sig- 
Table 1. Baseline Characteristics of Studies Included in the Network Meta-Analysis

\begin{tabular}{|c|c|c|c|c|c|c|c|c|c|}
\hline $\begin{array}{l}\text { Author } \\
\text { (year) }\end{array}$ & $\begin{array}{c}\text { Study } \\
\text { identifier }\end{array}$ & $\begin{array}{l}\text { Study } \\
\text { phase }\end{array}$ & $\begin{array}{l}\text { Treatment } \\
\text { phase }\end{array}$ & Study treatments & $\begin{array}{c}\text { Sample } \\
\text { size, } \\
n\end{array}$ & $\begin{array}{l}\text { Male } \\
\text { sex } \\
(\%)\end{array}$ & $\begin{array}{l}\text { Mean } \\
\text { age } \\
\text { (yr) }\end{array}$ & $\begin{array}{c}\text { Mean } \\
\text { disease } \\
\text { duration } \\
\text { (yr) }\end{array}$ & $\begin{array}{l}\text { Mean } \\
\text { Mayo } \\
\text { score }\end{array}$ \\
\hline \multirow{2}{*}{$\begin{array}{l}\text { Hibi et al. } \\
(2017)^{16}\end{array}$} & NCT01863771 & 3 & Maintenance & Placebo & 31 & 61 & 42.9 & $5.7^{\mathrm{a}}$ & $8.0^{\mathrm{a}}$ \\
\hline & (PURSUIT-J) & & & Golimumab (SC) $100 \mathrm{mg}$ Q4W & 32 & 59 & 39.3 & $5.4^{\mathrm{a}}$ & $8.0^{\mathrm{a}}$ \\
\hline \multirow{4}{*}{$\begin{array}{l}\text { Kobayashi } \\
\text { et al. }(2016)^{17}\end{array}$} & Japic CTI-060298 & 3 & Induction & Placebo & 104 & 64 & 37.8 & 7.1 & 8.5 \\
\hline & & & & Infliximab (IV) $5 \mathrm{mg} / \mathrm{kg}$ wk 0, 2, 6 & 104 & 63 & 40.0 & 8.1 & 8.6 \\
\hline & & & Maintenance & Placebo & 72 & NA & NA & NA & NA \\
\hline & & & & Infliximab (IV) 5 mg/kg wk 14, 22 & 73 & NA & NA & NA & NA \\
\hline \multirow{4}{*}{$\begin{array}{l}\text { Suzuki et al. } \\
(2014)^{19}\end{array}$} & NCT00853099 & $2 / 3$ & Induction & Placebo & 96 & 73 & 41.3 & 7.8 & 8.5 \\
\hline & & & & Adalimumab (SC) $160 \mathrm{mg}$ wk 0, $80 \mathrm{mg}$ wk 2 & 90 & 68 & 42.5 & 7.8 & 8.6 \\
\hline & & & Maintenance & Placebo & 96 & 73 & 41.3 & 7.8 & 8.5 \\
\hline & & & & Adalimumab (SC) $40 \mathrm{mg} 02 \mathrm{~W}$ & 177 & 63 & 43.4 & 8.0 & 8.6 \\
\hline \multirow{4}{*}{$\begin{array}{l}\text { Motoya et al. } \\
(2019)^{18}\end{array}$} & NCT02039505 & 3 & Induction & Placebo & 82 & 67 & 44.0 & 8.6 & 8.1 \\
\hline & & & & Vedolizumab (IV) 300 mg wk 0, 2, 6 & 164 & 60 & 42.3 & 7.2 & 8.3 \\
\hline & & & Maintenance & Placebo & 42 & 55 & 42.6 & 8.7 & 7.9 \\
\hline & & & & Vedolizumab (IV) 300 mg 08W & 41 & 51 & 43.0 & 8.6 & 8.1 \\
\hline
\end{tabular}

${ }^{2}$ Values expressed as median.

SC, subcutaneous; IV, intravenous; 02W, every 2 weeks; 04W, every 4 weeks; 08W, every 8 weeks; NA, not available.

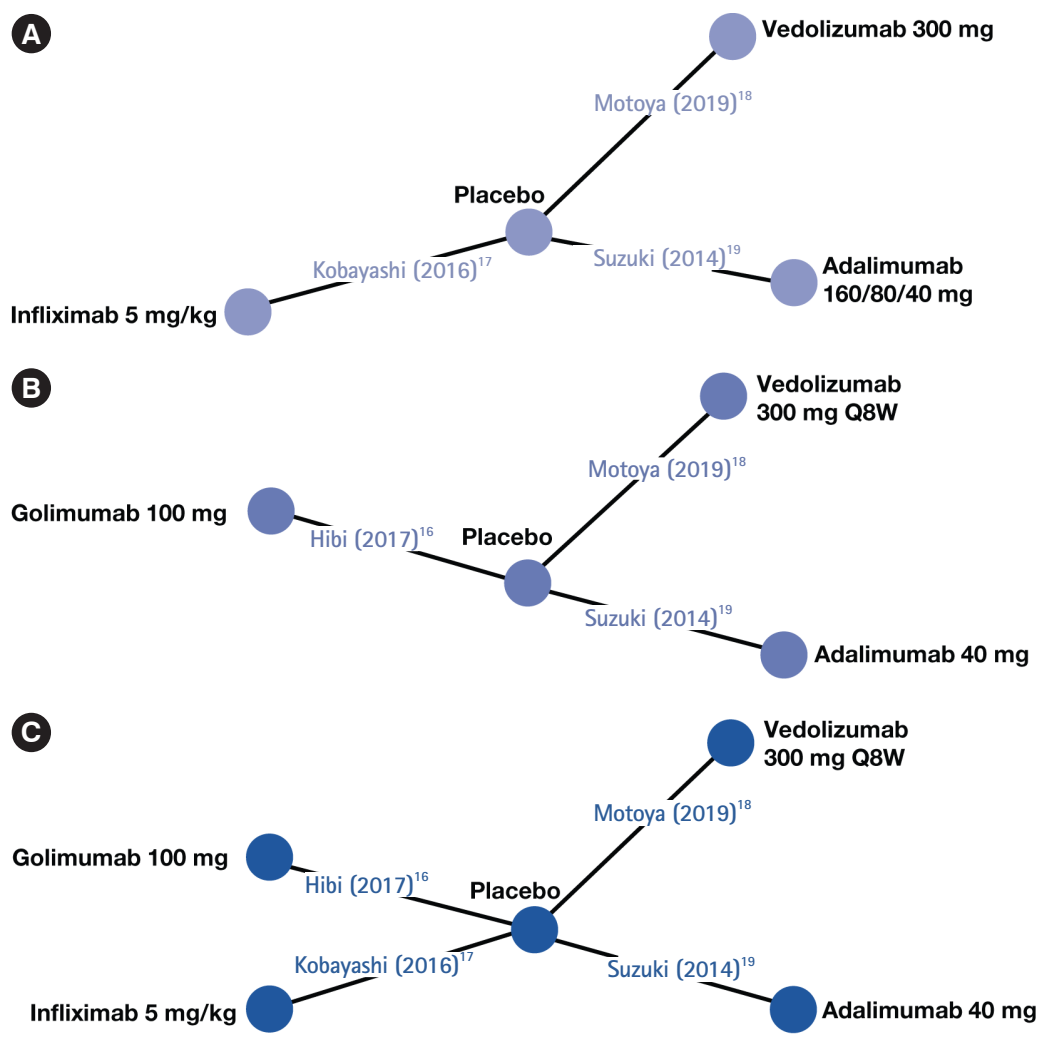

Fig. 1. Evidence structure of eligible comparisons for network meta-analysis. (A) Induction phase: response, remission, and mucosal healing (3 studies and 3 active treatments). (B) Maintenance phase: sustained response and sustained remission (3 studies and 3 active treatments). (C) Maintenance phase: mucosal healing ( 4 studies and 4 active treatments). 
Table 2. Probability of Efficacy Outcomes and Risk Differences with Active Treatments versus Placebo

\begin{tabular}{|c|c|c|c|c|c|c|}
\hline \multirow{2}{*}{$\begin{array}{l}\text { Induction phase } \\
\text { treatment }\end{array}$} & \multicolumn{2}{|c|}{ Clinical response } & \multicolumn{2}{|c|}{ Clinical remission } & \multicolumn{2}{|c|}{ Mucosal healing } \\
\hline & $\begin{array}{l}\text { Probability, } \\
\% \text { (95\% Crl) }\end{array}$ & $\begin{array}{c}\mathrm{RD}, \\
\%(95 \% \mathrm{Crl})\end{array}$ & $\begin{array}{l}\text { Probability, } \\
\%(95 \% \text { Crl) }\end{array}$ & $\begin{array}{c}\mathrm{RD}, \\
\%(95 \% \mathrm{Crl})\end{array}$ & $\begin{array}{l}\text { Probability, } \\
\% \text { (95\% Crl) }\end{array}$ & $\begin{array}{c}\text { RD, } \\
\%(95 \% \mathrm{Crl})\end{array}$ \\
\hline Placebo & 0.37 (0.31 to 0.43 ) & Reference & 0.11 (0.08 to 0.15$)$ & Reference & 0.30 (0.24 to 0.36$)$ & Reference \\
\hline Adalimumab 160/80 mg & 0.46 (0.34 to 0.59 ) & $0.09(-0.04$ to 0.23$)$ & 0.16 (0.09 to 0.25 ) & $0.05(-0.02$ to 0.14$)$ & 0.44 (0.31 to 0.58$)$ & 0.14 (0.00 to 0.29$)$ \\
\hline Infliximab 5 mg/kg & 0.55 (0.43 to 0.67$)$ & 0.18 (0.06 to 0.30$)$ & 0.22 (0.14 to 0.32$)$ & 0.11 (0.03 to 0.21$)$ & 0.49 (0.35 to 0.62$)$ & 0.19 (0.05 to 0.33$)$ \\
\hline \multirow[t]{3}{*}{ Vedolizumab $300 \mathrm{mg}$} & 0.55 (0.40 to 0.70$)$ & 0.18 (0.01 to 0.35$)$ & 0.22 (0.12 to 0.35$)$ & 0.11 (0.01 to 0.25 ) & 0.46 (0.31 to 0.63$)$ & $0.16(-0.02$ to 0.36 \\
\hline & \multicolumn{2}{|c|}{ Sustained clinical response } & \multicolumn{2}{|c|}{ Sustained clinical remission } & \multicolumn{2}{|c|}{ Mucosal healing } \\
\hline & $\begin{array}{l}\text { Probability, } \\
\%(95 \% \text { Crl) }\end{array}$ & $\begin{array}{c}\text { RD, } \\
\%(95 \% \text { Crl) } \\
\end{array}$ & $\begin{array}{l}\text { Probability, } \\
\%(95 \% \text { Crl) }\end{array}$ & $\begin{array}{c}\text { RD, } \\
\%(95 \% \text { Crl) } \\
\end{array}$ & $\begin{array}{l}\text { Probability, } \\
\%(95 \% \text { Crl) }\end{array}$ & $\begin{array}{c}\text { RD, } \\
\%(95 \% \text { Crl) } \\
\end{array}$ \\
\hline Placebo & 0.09 (0.05 to 0.15$)$ & Reference & 0.03 (0.01 to 0.06 ) & Reference & 0.11 (0.06 to 0.17 ) & Reference \\
\hline Adalimumab $40 \mathrm{mg}$ & 0.15 (0.06 to 0.30$)$ & $0.06(-0.03$ to 0.21$)$ & 0.06 (0.02 to 0.15$)$ & $0.06(-0.02$ to 0.11$)$ & 0.21 (0.10 to 0.36$)$ & 0.10 (0.02 to 0.23$)$ \\
\hline Golimumab 100 mg & 0.34 (0.17 to 0.58 ) & 0.25 (0.06 to 0.51 ) & 0.18 (0.07 to 0.38 ) & 0.14 (0.03 to 0.35 ) & 0.60 (0.27 to 0.91$)$ & 0.49 (0.13 to 0.85$)$ \\
\hline Infliximab 5 mg/kg & NA & NA & NA & NA & 0.21 (0.10 to 0.36$)$ & 0.10 (0.02 to 0.22$)$ \\
\hline Vedolizumab $300 \mathrm{mg}$ & 0.28 (0.13 to 0.47$)$ & 0.18 (0.04 to 0.38$)$ & 0.13 (0.05 to 0.28 ) & 0.10 (0.02 to 0.24$)$ & 0.31 (0.14 to 0.56$)$ & 0.20 (0.04 to 0.46$)$ \\
\hline
\end{tabular}

$\mathrm{RD}$, risk difference; $\mathrm{Crl}$, credible intervals; $\mathrm{NA}$, not available.

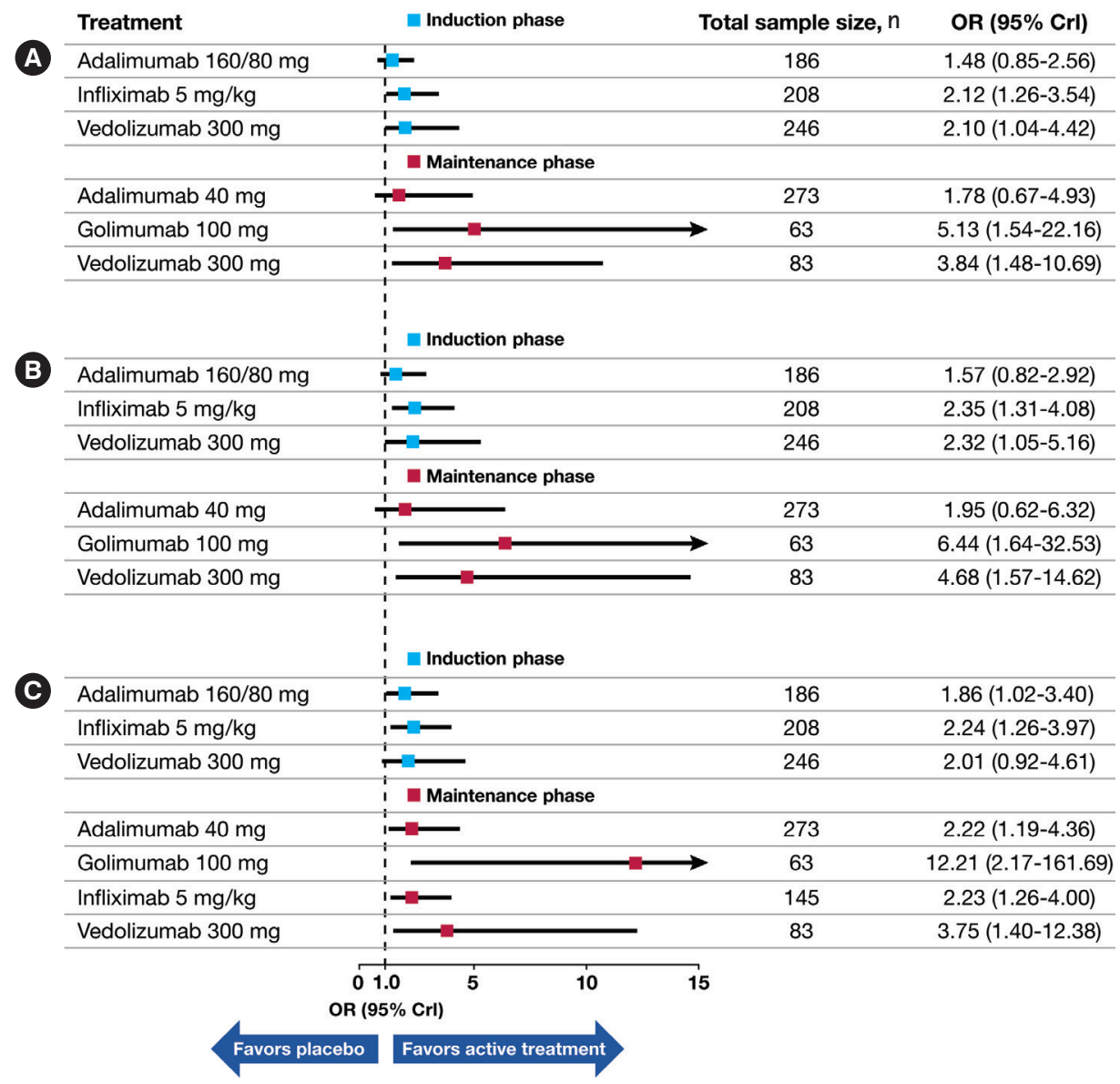

Fig. 2. Odds of efficacy outcomes with active treatments versus placebo. (A) Clinical response in induction and sustained response in maintenance. (B) Clinical remission in induction and sustained remission in maintenance. (C) Mucosal healing in induction and maintenance. $\mathrm{OR}$, odds ratio; $\mathrm{Crl}$, credible intervals. 
nificantly higher with adalimumab and infliximab (Table 2), with corresponding odds of 1.86 (95\% CrI, 1.02-3.40) and 2.24 (95\% CrI, 1.26-3.97), respectively (Fig. 2C).

\section{Maintenance Phase Efficacy Outcomes}

Treatment efficacy was examined using data from week 48 to week 60 in the 4 trials that reported outcomes following maintenance therapy with adalimumab, golimumab, infliximab, and vedolizumab (Fig. 1B and C).

Compared with placebo, the likelihood of sustained clinical response and sustained clinical remission with adalimumab was not significantly different. In contrast, compared with placebo, patients who received golimumab and vedolizumab had a higher probability of sustained response and sustained remission (Table 2). Odds of sustained clinical response were significantly higher with golimumab (OR, 5.13; 95\% CrI, 1.5422.16) and with vedolizumab (OR, 3.84; 95\% CrI, 1.48-10.69) (Fig. 2A).

Likewise, the odds of sustained clinical remission were significantly higher with golimumab (OR, 6.44; 95\% CrI, 1.6432.53 ) and with vedolizumab (OR, 4.68; 95\% CrI, 1.57-14.62) (Fig. 2B). Compared with placebo, the probability of mucosal healing was significantly higher with all biologics (Table 2). The odds of mucosal healing on maintenance therapy were adalimumab OR of 2.22 (95\% CrI, 1.19-4.36), golimumab OR of 12.21 (95\% CrI, 2.17-161.69), infliximab OR of 2.23 (95\% CrI, 1.26-4.00), and vedolizumab OR of 3.75 (95\% CrI, 1.4012.38) (Fig. 2C).

\section{DISCUSSION}

Genetic factors may contribute to UC pathogenesis and susceptibility, including loci identified in the Japanese population. ${ }^{20}$ Population-based factors may also be important in response to treatment. To our knowledge, this is the first NMA to evaluate the relative efficacy of biologics for UC approved in Japan among biologic-naïve Japanese patients with UC. Despite the limited number of studies and the small sample sizes with relevant data in the biologic-naïve Japanese population, our NMA provides insights into relative biologic efficacies in this specific subset of UC patients. Overall, biologic therapies showed varying efficacies during induction and maintenance; however, there was no evidence to suggest substantial differences, and efficacy outcomes were relatively similar among the assessed biologics. Compared with placebo, vedolizumab and infliximab had a similar high likelihood for achieving clini- cal response, remission, and mucosal healing during induction treatment, whereas golimumab and vedolizumab had the highest likelihood of maintaining these outcomes. During maintenance treatment, in comparison with placebo, golimumab and vedolizumab had the highest likelihood of maintaining efficacy outcomes.

The incidence of adverse events (AEs) associated with the induction treatment of the biologics versus placebo were similar (infliximab, $81.7 \%$ vs. $82.7 \%$; adalimumab, $44.4 \%$ vs. $46.9 \%$; vedolizumab, $50.0 \%$ vs. $52.4 \%$ ). Compared with placebo, fewer patients had serious AEs, serious infections, or worsening of disease in the induction treatment of infliximab (8.7\% vs. $12.5 \%, 1.0 \%$ vs. $1.9 \%, 7.7 \%$ vs. $10.6 \%$, respectively). In contrast, more patients experienced AEs with the maintenance treatment of golimumab (97.0\% vs. $71.0 \%$ ), infliximab (96.2\% vs. 90.4\%), adalimumab (538 events vs. 273 events), and vedolizumab ( $87.8 \%$ vs. $78.6 \%)$. Adalimumab also resulted in a higher number of serious AEs (33 events vs. 14 events), serious infections (8 events vs. 2 events), and worsening of disease (18 events vs. 15 events) than placebo. There were no substantial differences with infliximab in serious AEs (17.3\% vs. 18.3\%), serious infections (1.0\% vs. 1.9\%), and worsening of disease ( $15.4 \%$ vs. $17.3 \%$ ) or with vedolizumab in serious AEs (9.8\% vs. $7.1 \%$ ). Malignancy was reported in 2 patients (pancreatic carcinoma and parathyroid tumor) treated with adalimumab. No available data on malignancy were noted on the other drugs.

In a previous NMA evaluating treatment efficacies in a global population of anti-TNF therapy-naïve UC patients, all analyzed treatments (tofacitinib, adalimumab, golimumab, infliximab, and vedolizumab) were superior to placebo for both induction and maintenance treatment of UC. ${ }^{21}$ In this report, a traditional meta-analysis of outcomes data for each treatment demonstrated similar higher odds of clinical remission, clinical response, and mucosal healing with infliximab (OR: 3.62, 3.97 , and 3.05, respectively) followed by vedolizumab (OR: $3.17,4.26,2.91$, respectively). These results are consistent with results from our indirect treatment comparisons, wherein the induction-phase NMA showed that infliximab treatment was better than adalimumab and golimumab in achieving clinical response, better than adalimumab in achieving clinical remission, and better than adalimumab and golimumab in achieving mucosal healing. No other indirect comparisons reached statistical significance. Because of the differences in study design (patient eligibility for maintenance phase; rerandomization of induction responders), indirect treatment comparisons with maintenance-phase data were not conducted. 
Another NMA of global population data in anti-TNF therapy-naïve UC patients also found that all biologics (adalimumab, golimumab, infliximab, and vedolizumab) were more effective at inducing clinical response, remission, and mucosal healing in induction. ${ }^{22}$ In this study, data from both rerandomization trials and straight trials were included in indirect treatment comparisons of maintenance-phase data. Any study design differences were accounted for by assuming the number of responders at end of induction to be equivalent to the number rerandomized. In the maintenance phase, however, only vedolizumab showed significantly better odds across all 3 efficacy outcomes: sustained clinical response, remission, and mucosal healing. In indirect treatment comparisons, vedolizumab showed significantly better durable clinical response than adalimumab, infliximab, and golimumab during maintenance. Vedolizumab also showed a significant improvement in clinical remission over infliximab and significant improvement in mucosal healing over adalimumab.

The recently reported VARSITY trial (NCT02497469) is the first and only published head-to-head RCT comparing the efficacy and safety of 2 biologic therapies, vedolizumab, and adalimumab, in patients with moderately to severely active UC. ${ }^{23}$ The lack of similar comparisons of biologic therapies in head-to-head trials for patients with UC, and in particular patient subpopulations of interest, highlights the importance of performing NMA studies.

Our NMA had several limitations. First, there are few published randomized controlled clinical trials of UC therapies specific to the biologic-naïve Japanese population. Thus, the currently available highest-level clinical evidence base is limited. As a result, comparative efficacy of tofacitinib could not be evaluated as tofacitinib efficacy data in a Japanese subpopulation were based on a small subset of the OCTAVE trials ${ }^{24}$ and thus not powered to detect differences. Second, because of the lack of head-to-head trials, efficacy analyses were based on indirect comparisons. Outcomes of this NMA should be assessed with consideration for the different study designs of each trial. Third, the lack of patient-level data also precluded accounting for differences in patient-related effect modifiers in individual trials (e.g., severity of UC); future analyses should control for such differences. In addition, the relatively small patient sample sizes available for inclusion in the current analyses were a limitation. A safety analysis also could not be conducted due to the small sample sizes and limited available data. Finally, differences in efficacy outcomes in the placebo arms from individual trials may have affected the results of this analysis. For example, the higher efficacy rates in Japanese patients who received placebo in the vedolizumab trial ${ }^{18}$ may have influenced the current NMA results.

In conclusion, among Japanese patients with UC, all biologic therapies evaluated showed superior efficacy relative to placebo, although the degree of efficacy benefit varied between drugs. Vedolizumab and infliximab had the highest likelihood for induction of clinical response, clinical remission, and mucosal healing, whereas golimumab had the highest likelihood of maintaining these outcomes. These results are not generalizable to other patient populations and require confirmation in larger patient populations and real-world settings. Future studies should also evaluate safety in addition to efficacy to determine the relative net benefit of biologic therapies.

\section{ADDITIONAL INFORMATION}

\section{Funding Source}

This study was sponsored by Takeda.

\section{Conflict of Interest}

Hibi T has received grants from AbbVie, EA Pharmaceutical, JIMRO, Otsuka Holdings, and Zeria Pharmaceutical; lecture fees from Aspen Japan KK, AbbVie GK, Ferring, Gilead Sciences, Janssen, JIMRO, Kisse Pharmaceutical, Mitsubishi-Tanabe Pharmaceutical, Mochida Pharmaceutical, Nippon Kayaku, Pfizer, Takeda Pharmaceutical, and Zeria Pharmaceutical. Kamae I has received research grants from Takeda, Crecon Medical Assessment, Ono Pharmaceutical Co, Ltd, and Becton, Dickinson and Company Japan; traveling expense compensated by Takeda to attend the Takeda global advisory meeting (to Istanbul, once). Iwakiri R and Ursos L are employed by Takeda. Patel H was an employee of Takeda at the time the research was performed. Hather G and Pinton P are employed by and own stocks in Takeda. But, no other potential conflict of interest relevant to this article was reported.

Hibi T is an editorial board member of the journal but did not involve in the peer reviewer selection, evaluation, or decision process of this article. No other potential conflicts of interest relevant to this article were reported.

\section{Author Contribution}

Study concept and design: Pinton P, Ursos L, Patel H. Literature review: Pinton P, Ursos L. Interpretation of data and adding clinical context: Hibi T, Kamae I, Pinton P, Ursos L, Iwakiri 
R, Hather G, Patel H. Drafting of the manuscript: Hibi T, Kamae I, Pinton P, Ursos L, Iwakiri R, Hather G, Patel H. All authors critically reviewed and revised the manuscript for important intellectual content and approved the final version of the manuscript before submission.

\section{Non-Author Contribution}

We thank Keith Chan of Precision Xtract (Vancouver, BC, Canada) for data analyses.

\section{ORCID}

Hibi T

https://orcid.org/0000-0002-6256-1204

Kamae I https://orcid.org/0000-0002-3191-0942

Pinton P https://orcid.org/0000-0002-4227-7687

Ursos L https://orcid.org/0000-0002-8109-0188

Iwakiri R

Hather G https://orcid.org/0000-0001-7911-9669

Patel H https://orcid.org/0000-0003-3868-8309 https://orcid.org/0000-0002-1827-5163

\section{Supplementary Material}

Supplementary materials are available at the Intestinal Research website (https://www.irjournal.org).

\section{REFERENCES}

1. Ungaro R, Mehandru S, Allen PB, Peyrin-Biroulet L, Colombel JF. Ulcerative colitis. Lancet 2017;389:1756-1770.

2. Yarlas A, Rubin DT, Panés J, et al. Burden of ulcerative colitis on functioning and well-being: a systematic literature review of the SF-36" health survey. J Crohns Colitis 2018;12:600-609.

3. Ueno F, Nakayama Y, Hagiwara E, Kurimoto S, Hibi T. Impact of inflammatory bowel disease on Japanese patients' quality of life: results of a patient questionnaire survey. J Gastroenterol 2017;52:555-567.

4. Ng SC, Tsoi KK, Kamm MA, et al. Genetics of inflammatory bowel disease in Asia: systematic review and meta-analysis. Inflamm Bowel Dis 2012;18:1164-1176.

5. Japanese Ministry of Health Labour and Welfare. Handbook of Health and Welfare Statistics 2017: Table 2-22 [Internet]. c2019 [cited 2019 Sep 24]. https://www.mhlw.go.jp/english/ database/db-hh/2-1.html.

6. Harbord M, Eliakim R, Bettenworth D, et al. Third European evidence-based consensus on diagnosis and management of ulcerative colitis. Part 2: current management. J Crohns Colitis 2017;11:769-784.

7. Soler D, Chapman T, Yang LL, Wyant T, Egan R, Fedyk ER. The binding specificity and selective antagonism of vedolizumab, an anti-alpha4beta7 integrin therapeutic antibody in development for inflammatory bowel diseases. J Pharmacol Exp Ther 2009;330:864-875.

8. Pharmaceuticals and Medical Devices Agency. New drugs approved in July 2018 [Internet]. c2019 [cited 2019 Jun 18]. https://www.pmda.go.jp/files/000229080.pdf\#page=2.

9. Okabayashi S, Kobayashi T, Hibi T. Drug lag for inflammatory bowel disease treatments in the East and West. Inflamm Intest Dis 2018;3:25-31.

10. Peyrin-Biroulet L, Sandborn W, Sands BE, et al. Selecting Therapeutic Targets in Inflammatory Bowel Disease (STRIDE): determining therapeutic goals for treat-to-target. Am J Gastroenterol 2015;110:1324-1338.

11. Liu JZ, van Sommeren S, Huang H, et al. Association analyses identify 38 susceptibility loci for inflammatory bowel disease and highlight shared genetic risk across populations. Nat Genet 2015;47:979-986.

12. Ng WK, Wong SH, Ng SC. Changing epidemiological trends of inflammatory bowel disease in Asia. Intest Res 2016;14:111119.

13. Hoaglin DC, Hawkins N, Jansen JP, et al. Conducting indirecttreatment-comparison and network-meta-analysis studies: report of the ISPOR Task Force on Indirect Treatment Comparisons Good Research Practices. Part 2. Value Health 2011; 14:429-437.

14. Higgins JP, Altman DG, Gøtzsche PC, et al. The Cochrane Collaboration's tool for assessing risk of bias in randomised trials. Version 2. BMJ 2011;343:d5928.

15. Mantel N, Haenszel W. Statistical aspects of the analysis of data from retrospective studies of disease. J Natl Cancer Inst 1959;22:719-748.

16. Hibi T, Imai Y, Senoo A, Ohta K, Ukyo Y. Efficacy and safety of golimumab 52-week maintenance therapy in Japanese patients with moderate to severely active ulcerative colitis: a phase 3, double-blind, randomized, placebo-controlled study(PURSUIT-J study). J Gastroenterol 2017;52:1101-1111.

17. Kobayashi T, Suzuki Y, Motoya S, et al. First trough level of infliximab at week 2 predicts future outcomes of induction therapy in ulcerative colitis-results from a multicenter prospective randomized controlled trial and its post hoc analysis. J Gastroenterol 2016;51:241-251.

18. Motoya S, Watanabe $\mathrm{K}$, Ogata $\mathrm{H}$, et al. Vedolizumab in Japanese patients with ulcerative colitis: a phase 3, randomized, double-blind, placebo-controlled study. PLoS One 2019;14: e0212989. 
19. Suzuki Y, Motoya S, Hanai H, et al. Efficacy and safety of adalimumab in Japanese patients with moderately to severely active ulcerative colitis. J Gastroenterol 2014;49:283-294.

20. Asano K, Matsushita T, Umeno J, et al. A genome-wide association study identifies three new susceptibility loci for ulcerative colitis in the Japanese population. Nat Genet 2009;41: 1325-1329.

21. Bonovas S, Lytras T, Nikolopoulos G, Peyrin-Biroulet L, Danese S. Systematic review with network meta-analysis: comparative assessment of tofacitinib and biological therapies for moderate-to-severe ulcerative colitis. Aliment Pharmacol Ther 2018;47:454-465.
22. Vickers AD, Ainsworth C, Mody R, et al. Systematic review with network meta-analysis: comparative efficacy of biologics in the treatment of moderately to severely active ulcerative colitis. PLoS One 2016;11:e0165435.

23. Sands BE, Peyrin-Biroulet L, Loftus EV Jr, et al. Vedolizumab versus adalimumab for moderate-to-severe ulcerative colitis. N Engl J Med 2019;381:1215-1226.

24. Motoya S, Watanabe M, Kim HJ, et al. Tofacitinib induction and maintenance therapy in East Asian patients with active ulcerative colitis: subgroup analyses from three phase 3 multinational studies. Intest Res 2018;16:233-245. 


\section{See "Efficacy of biologic therapies for biologic-naïve Japanese patients with moderately to severely active ulcerative colitis: a network meta-analysis" on page 53-61.}

Supplementary Table 1. Outcome Measures and Definitions

\begin{tabular}{|c|c|c|c|c|}
\hline Efficacy outcome & Hibi et al. (2017) ${ }^{1}$ & Kobayashi et al. (2016) ${ }^{2}$ & Motoya et al. (2019) ${ }^{3}$ & Suzuki et al. (2014) ${ }^{4}$ \\
\hline \multicolumn{5}{|l|}{ Induction phase } \\
\hline Clinical response & - & $\begin{array}{l}\text { Clinical response (defined as } \\
\text { a decrease in the total Mayo } \\
\text { baseline score of at least } 3 \\
\text { points and at least } 30 \% \text {, with } \\
\text { an accompanying decrease in } \\
\text { the rectal bleeding subscore of } \\
\text { at least } 1 \text { point or an absolute } \\
\text { subscore of } 0 \text { or } 1 \text { ) at wk } 8\end{array}$ & $\begin{array}{l}\text { Clinical response (defined as } \\
\text { a reduction of } \geq 3 \text { points } \\
\text { and } \geq 30 \% \text { from baseline } \\
\text { in the full Mayo score and } \\
\text { a } \geq 1 \text {-point decrease in the } \\
\text { rectal bleeding subscore or } \\
\text { an absolute rectal bleeding } \\
\text { subscore } \leq 1 \text { ) at wk } 10\end{array}$ & $\begin{array}{l}\text { Clinical response (defined } \\
\text { as a full Mayo score } \\
\text { decrease of } \geq 3 \text { points and } \\
\geq 30 \% \text { from baseline plus } \\
\text { a decrease in the rectal } \\
\text { bleeding subscore [RBS] } \\
\geq 1 \text { or an absolute RBS of } \\
\leq 1 \text { ) at wk } 8\end{array}$ \\
\hline Clinical remission & - & $\begin{array}{l}\text { Clinical remission (defined as a } \\
\text { total Mayo score of } 2 \text { points } \\
\text { or lower, with no individual } \\
\text { subscore exceeding } 1 \text { point) at } \\
\text { wk } 8\end{array}$ & $\begin{array}{l}\text { Clinical remission (defined as } \\
\text { a full Mayo score } \leq 2 \text { and } \\
\text { no subscore }>1 \text { ) at wk } 10\end{array}$ & $\begin{array}{l}\text { Clinical remission (defined as } \\
\text { a full Mayo score } \leq 2 \text { with } \\
\text { no individual subscore }>1 \text { ) } \\
\text { at wk } 8\end{array}$ \\
\hline Mucosal healing & - & $\begin{array}{l}\text { Mucosal healing (defined as an } \\
\text { endoscopic subscore of } 0 \text { or } 1 \text { ) } \\
\text { at wk } 8\end{array}$ & $\begin{array}{l}\text { Mucosal healing (defined as } \\
\text { an endoscopic subscore } \\
\leq 1 \text { ) at wk } 10\end{array}$ & $\begin{array}{l}\text { Mucosal healing (defined } \\
\text { as an endoscopy subscore } \\
\leq 1 \text { ) at wk } 8\end{array}$ \\
\hline \multicolumn{5}{|l|}{ Maintenance phase } \\
\hline $\begin{array}{l}\text { Sustained clinical } \\
\text { response }\end{array}$ & $\begin{array}{l}\text { Maintenance of clinical response } \\
\text { (defined as a decrease in the } \\
\text { Mayo score by } \geq 30 \% \text { and } \geq 3 \\
\text { points from induction wk } 0 \text {, } \\
\text { along with a fall in the rectal } \\
\text { bleeding subscore of } \geq 1 \text { or a } \\
\text { rectal bleeding subscore of } 0 \\
\text { or } 1 \text { ) through wk } 54\end{array}$ & - & $\begin{array}{l}\text { Durable clinical response } \\
\text { (defined as a clinical } \\
\text { response at both wk } 10 \\
\text { and } 60 \text { ) }\end{array}$ & $\begin{array}{l}\text { Clinical response at wk } 8 \\
\text { and wk } 52\end{array}$ \\
\hline $\begin{array}{l}\text { Sustained clinical } \\
\text { remission }\end{array}$ & $\begin{array}{l}\text { Clinical remission (defined as a } \\
\text { Mayo score of } \leq 2 \text { points, with } \\
\text { no individual subscore }>1 \text { ) at } \\
\text { both maintenance-wk } 30 \text { and } \\
\text { maintenance-wk } 54\end{array}$ & - & $\begin{array}{l}\text { Durable remission (defined } \\
\text { as having clinical remission } \\
\text { at both wk } 10 \text { and } 60 \text { ) }\end{array}$ & $\begin{array}{l}\text { Clinical remission at wk } 8 \\
\text { and wk } 52\end{array}$ \\
\hline Mucosal healing & $\begin{array}{l}\text { Mucosal healing (defined as } \\
\text { measured using a Mayo } \\
\text { endoscopic subscore of } 0 \text { or } \\
\text { 1) at both maintenance-wk } 30 \\
\text { and maintenance-wk } 54\end{array}$ & Mucosal healing at wk 30 & Mucosal healing at wk 60 & Mucosal healing at wk 52 \\
\hline
\end{tabular}

1. Hibi T, Imai Y, Senoo A, Ohta K, Ukyo Y. Efficacy and safety of golimumab 52-week maintenance therapy in Japanese patients with moderate to severely active ulcerative colitis: a phase 3, double-blind, randomized, placebo- controlled study-(PURSUIT-J study). J Gastroenterol 2017;52:1101-1111.

2. Kobayashi T, Suzuki Y, Motoya S, et al. First trough level of infliximab at week 2 predicts future outcomes of induction therapy in ulcerative colitisresults from a multicenter prospective randomized controlled trial and its post hoc analysis. J Gastroenterol 2016;51:241-251.

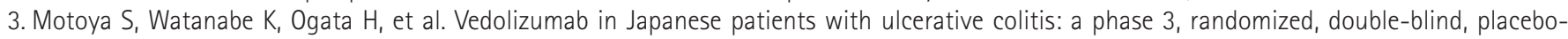
controlled study. PLoS One 2019;14:e0212989.

4. Suzuki Y, Motoya S, Hanai $H_{1}$ et al. Efficacy and safety of adalimumab in Japanese patients with moderately to severely active ulcerative colitis. J Gastroenterol 2014;49:283-294. 
Supplementary Table 2. Cochrane Assessment of Bias Risk of Randomized Controlled Trials

\begin{tabular}{|c|c|c|c|c|}
\hline Bias domain & Hibi et al. (2017) & Kobayashi et al. (2016) ${ }^{2}$ & Suzuki et al. $(2014)^{3}$ & Motoya et al. (2019) \\
\hline Randomization sequence generation & Low & Low & Low & Low \\
\hline Allocation concealment & Unclear & Unclear & Unclear & Unclear \\
\hline Blinding of participants and personnel & Low & Low & Low & Low \\
\hline Blinding of outcome assessment & Low & Low & Low & Low \\
\hline Incomplete outcome data & Low & Low & Low & Low \\
\hline Selective outcome reporting & Low & Low & Low & Low \\
\hline Other sources of bias & Low & Low & Low & Low \\
\hline Overall bias risk & Low & Low & Low & Low \\
\hline
\end{tabular}

1. Hibi T, Imai Y, Senoo A, Ohta K, Ukyo Y. Efficacy and safety of golimumab 52-week maintenance therapy in Japanese patients with moderate to severely active ulcerative colitis: a phase 3, double-blind, randomized, placebo- controlled study-(PURSUIT-J study). J Gastroenterol 2017;52:1101-1111.

2. Kobayashi T, Suzuki Y, Motoya S, et al. First trough level of infliximab at week 2 predicts future outcomes of induction therapy in ulcerative colitisresults from a multicenter prospective randomized controlled trial and its post hoc analysis. J Gastroenterol 2016;51:241-251.

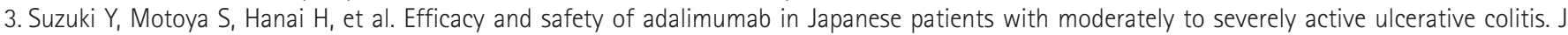
Gastroenterol 2014;49:283-294.

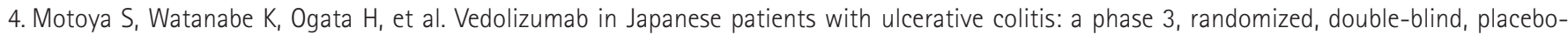
controlled study. PLoS One 2019;14:e0212989. 